\title{
Fusion-oncogenes are associated with increased metastatic capacity and persistent disease
} in pediatric thyroid cancers

Aime T. Franco, $\mathrm{PhD}^{1 \#}$, Julio C. Ricarte-Filho, $\mathrm{PhD}^{1 \#}$, Amber Isaza ${ }^{1}$, Zachary Jones ${ }^{1}$, Neil Jain ${ }^{1}$, Sogol Mostoufi-Moab, MD, MSCE ${ }^{1,2}$, Lea Surrey, $\mathrm{MD}^{3}$, Theodore W. Laetsch, $\mathrm{MD}^{2}$, Marilyn M. $\mathrm{Li}, \mathrm{MD}^{3}$, Jessica Clague DeHart, $\mathrm{PhD}^{4}$, Erin Reichenberger, $\mathrm{PhD}^{5}$, Deanne Taylor, $\mathrm{PhD}^{5}$, Ken Kazahaya, MD, $\mathrm{MBA}^{6,7}$, N. Scott Adzick, $\mathrm{MD}^{7}$, and Andrew J. Bauer, $\mathrm{MD}^{1}$

${ }^{1}$ Division of Endocrinology and Diabetes, Children's Hospital of Philadelphia, Philadelphia, PA;

${ }^{2}$ Division of Oncology, Children's Hospital of Philadelphia, Philadelphia, PA; ${ }^{3}$ Department of Pathology and Laboratory Medicine, Children's Hospital of Philadelphia, Philadelphia, PA; ${ }^{4}$ School of Community and Global Health, Claremont Graduate University, Claremont, CA;

${ }^{5}$ Department of Biomedical and Health Informatics, Children's Hospital of Philadelphia, Philadelphia, PA; 'Division of Pediatric Otolaryngology, Children's Hospital of Philadelphia, Philadelphia, PA; ${ }^{7}$ Department of Surgery, Children's Hospital of Philadelphia, Philadelphia, PA. \#Contributed equally to this study

\section{Corresponding Author:}

Andrew J Bauer, MD

The Thyroid Center, Division of Endocrinology and Diabetes, Children's Hospital of

Philadelphia

3500 Civic Center Boulevard, Buerger Center for Advanced Pediatric Care 12-149, Philadelphia,

PA 19104

Tel: 215-590-5129

Email: bauera@chop.edu

Running Head: Classifying Oncogenic Variants in Pediatric Thyroid Cancer 


\author{
Abbreviations \\ CHOP The Children's Hospital of Philadelphia \\ CCFP CHOP Cancer Fusion Panel \\ CNA Copy Number Alteration \\ CSTP CHOP Solid Tumor Panel \\ cmvPTC Cribriform-Morular Variant Papillary Thyroid Cancer \\ cPTC Classic Papillary Thyroid Cancer \\ DTC Differentiated Thyroid Cancer \\ dsvPTC Diffuse Sclerosing Variant Papillary Thyroid Cancer \\ ETE Extra Thyroidal Extension \\ FNA Fine Needle Aspiration \\ FTC Follicular Thyroid Cancer \\ fvPTC Follicular Variant Papillary Thyroid Cancer \\ fvPTMC Follicular Variant Papillary Thyroid Microcarcinoma \\ LNM Lymph Node Metastasis \\ NGS Next Generation Sequencing \\ PCR Polymerase Chain Reaction \\ SNV Single-Nucleotide Variation \\ svPTC Solid Variant Papillary Thyroid Cancer \\ tcvPTC Tall Cell Variant Papillary Thyroid Cancer \\ WLPTC Warthin-like Papillary Thyroid Cancer
}




\section{ABSTRACT}

Background: In 2014, data from a comprehensive multiplatform analysis of 496 adult papillary thyroid cancer samples reported by The Cancer Genome Atlas project suggested that reclassification of thyroid cancer into molecular subtypes, $R A S$-like and $B R A F$-like, better reflects clinical behavior than sole reliance on pathological classification. The aim of this study was to categorize the common oncogenic variants in pediatric differentiated thyroid cancer and investigate if mutation subtype classification correlated with the risk of metastasis and response to initial therapy in pediatric DTC.

Methods: Somatic cancer gene panel analysis was completed on DTC from 131 pediatric patients. DTC were categorized into $R A S$-mutant $(H-K-N R A S), B R A F$-mutant (BRAF p.V600E) and RET/NTRK fusion (RET, NTRK1 and NTRK3 fusions) to determine differences between subtype classification in regard to pathological data (AJCC TNM) as well as response to therapy 1-year after initial treatment had been completed.

Results: Mutation-based subtype categories were significant in most variables, including age at diagnosis, metastatic behavior, and the likelihood of remission at 1-year. Patients with RET/NTRK fusions were significantly more likely to have advanced lymph node and distant metastasis and less likely to achieve remission at one year than patients within $R A S$ - or BRAF-mut subgroups.

Conclusions: Our data supports that genetic subtyping of pediatric DTC more accurately reflects clinical behavior than sole reliance on pathological classification with patients with RET/NTRK fusions having worse outcomes than those with $B R A F$-mutant disease. Future trials should consider inclusion of molecular subtype into risk stratification. 


\section{BACKGROUND}

In 2014, the Thyroid Cancer Genome Atlas (TCGA) reported that classification of adult PTC into molecular subtypes based on a mRNA expression signature, $R A S$-like and $B R A F$-like, more accurately reflected cellular signaling, cellular differentiation and clinical behavior when compared to histology alone. ${ }^{1}$ This observation has fueled debate whether identification of oncogenic alterations more accurately predict the risk of malignancy in thyroid nodules with indeterminate cytology may be used to stratify therapy, including the extent of surgery, lobectomy versus total thyroidectomy, as well as central compartment lymph node dissection. ${ }^{2,3}$

With the reduced costs of next-generation sequencing, the use of somatic cancer gene panel analysis in pediatric patients with differentiated thyroid cancer (DTC) has expanded with current data showing a shifted distribution of driver alterations with a higher incidence of oncogenic fusions rather than point mutations in children and adolescents compared to adults. ${ }^{4,5}$ Similar to the TCGA based molecular sub-type classification system based on mRNA expression signatures, we sought to determine whether oncogenic subtyping based on identified mutations or fusions predicts phenotypic behavior and outcomes in pediatric patients with DTC.

\section{METHODS}

\section{Patient population}

Our series comprised 131 pediatric thyroid tumors (122 PTCs and 9 FTCs) from surgical specimens. This included 66 surgical specimens sequentially collected from 2016-2019 and analyzed in the Department of Pathology at Children's Hospital of Philadelphia (CHOP) as well as 65 archived surgical specimens collected from 1989-2012 that were previously genotyped on a commercial platform. ${ }^{6}$ All of the tumors were sequentially collected and analyzed without bias 
selection limited only by sample adequacy. The research protocol was approved by CHOP's Institutional Review Board.

DTC classification was based on standard histopathological criteria defined by the World Health Organization. ${ }^{7}$ Tumors were staged according to the $7^{\text {th }}$ edition of the American Joint Committee on Cancer (AJCC) staging manual. ${ }^{8}$ Invasion was defined as spread to regional lymph nodes and/or distant metastasis. Remission was assessed at one year after surgery and defined as a basal-thyroglobulin ( $\mathrm{Tg}$ ) below the lower limit of detection and no evidence of persistent thyroid cancer on radiological or radioiodine whole-body scan (RAI-WBS). Neck ultrasound was used for patients with disease limited to the neck and undetectable Tg. Chest CT was added for patients with a history of pulmonary metastasis on initial imaging. RAI-WBS was used to assess for persistent disease in patients with detectable $\mathrm{Tg}(>10 \mathrm{ng} / \mathrm{ml}$ and/or increasing trend $)$ and no evidence of persistent thyroid cancer based on neck US and chest CT.

\section{Sequencing platform and variant calling}

The 66 tumors collected between 2016 and 2019 were sequenced as part of routine clinical care using both the CHOP Solid Tumor Panel (CSTP) and CHOP Cancer Fusion Panel (CCFP). CSTP is a targeted next-generation sequencing (NGS) assay encompassing 238 cancer genes. The assay is designed to detect single nucleotide variants (SNVs), indels, and copy number alterations (CNAs) as described previously. ${ }^{9}$ Briefly, genomic DNA was extracted from the tumor samples and libraries were prepared using probes targeting 238 genes and sequenced on HiSeq platform using $150 \mathrm{bp}$ paired-end sequencing. Sequence data were analyzed using the home brew software ConcordS v2 (for SNVs and indels) and NextGENe v2 NGS Analysis Software (for CNAs; SoftGenetics, LLC, State College, PA). Fusion gene detection was performed using the CHOP Cancer Fusion Panel as previously described. ${ }^{10}$ Briefly, target-specific primers covering 673 exons 
were custom designed to identify known fusion genes and potential novel fusion genes associated with 110 cancer genes using Anchored Multiplex PCR (AMPTM) technology (ArcherDX, Inc. Boulder, CO). The 65 cases collected between 1989 and 2012 had been previously genotyped using Asuragen's first-generation thyroid test, miRinform ${ }^{\circledR}$ Thyroid Test, as described. ${ }^{6}$ This panel interrogates the presence of the most common mutations in BRAF, HRAS, KRAS and NRAS and three fusion transcripts (RET/PTC1, RET/PTC3 and PAX8/PPARG). The miRinform ${ }^{\circledR}$ panel did not include analysis for DICER 1 mutations or NTRK-fusions. Unfortunately, tissue from the miRinform ${ }^{\circledR}$ cohort was not available for repeat analysis using the more comprehensive CHOP panels. All cancer genes included in the CSTP and CCFP panels and the miRInform Thyroid Test are listed in Supplementary Table 1.

Mutations were subcategorized into three groups, $R A S$-mutant $(H / K / N R A S$ mutations and PAX8/PPARG fusions), BRAF-mutant (BRAF p.V600E mutations), or RET/NTRK fusions (RET, NTRK1 and NTRK3 fusions) based on previous published reports in adults ${ }^{1,2,11}$ as well as pediatric data showing genotype-associated differences in invasive behavior. ${ }^{12-14}$ Tumors with no identified genetic alteration in the miRInform Thyroid Test, or genetic alterations classified as not previously identified as driver mutations in pediatric DTC with the CSTP panel, were characterized as indeterminate. $^{9}$

\section{Data analyses}

Data analysis was performed using Statistical analyses were run in R 4.0.5 and R Studio 1.4.1106. Frequencies and proportions were used as descriptive statistics for categorical variables. Mutation status was the primary variable of interest and so this was explored over a number of different dimensions of the data. Associations between covariates and mutation status were tested 
using Fisher's exact tests, to account for small cell sizes. A two-sided p value of less than 0.05 was considered statistically significant. Mutation data and clinicopathological characteristics from adult papillary thyroid cancer was collected from The Cancer Genome Atlas (TCGA) Data Portal (https://tcga-data.nci.nih.gov).

\section{RESULTS}

Patient Demographics and Thyroid Pathology

Clinicopathologic characteristics of the study population are summarized in Table 1 . The study cohort included 131 samples from 97 (74\%) female patients and 34 (26\%) male patients with a mean age of $14.53 \pm 2.99$ years. Tumors were divided based on their histopathological features: 60 (45.8\%) classic variant (cPTC), 29 (22.1\%) follicular variant (fvPTC), 14 (10.7\%) diffuse sclerosing variant (dsvPTC), 8 (6.1\%) mixed cPTC and fvPTC, 11 (8.4\%) were other forms of PTC, including cribriform-morular variant (cmvPTC), solid variant (svPTC), and Warthin-like (WLPTC), and 9 (6.9\%) follicular thyroid carcinoma (FTC).

\section{Identification and grouping of genetic alterations.}

Of the 131 patient samples, genetic alterations were identified in 78 of the 131 tumors; 9 with a $R A S$-mutant $(6.9 \%, 9 / 131), 26$ with $B R A F(19.8 \%, 26 / 131)$, and 43 with a $R E T$ - or NTRKfusion (32.8\%, 43/131; Table 1). There were 53 tumors classified as indeterminate. Secondary to more limited coverage of oncogenic driver alterations, there were more tumors classified as indeterminate in the miRInform group compared to the CSTP/CCFP group $(62.3 \%$ vs $37.7 \%)$ most notably fewer RET/NTRK-fusions identified in the miRInform group compared to the CSTP/CCFP group (27.9\% vs 72.1\%; Table 1). 
Figure 1 summarizes the mutational landscape of pediatric DTC and clinicopathological features. As previously reported, we found a correlation between genotype and pathological phenotype. ${ }^{12-14} R A S$-mutations and $P A X 8-P P A R G$ fusions were more commonly associated with fvPTC and FTCs than the other genotypes. There were 4 NRAS p.Q61R and $1 P A X 8-P P A R G$ fusions in five encapsulated fvPTC, one NRAS p.Q61R in a cPTC, one $P A X 8 / P P A R G$ in a cPTC/fvPTC/svPTC mixed histology and a single HRAS p.Q61R and single KRAS p.G12V in two FTC samples. The BRAF p.V600E mutation was most commonly associated with cPTC, observed in $26(19.8 \%)$ samples. RET/NTRK fusions were found in 43 (32.8\%) tumor samples spread across various subtypes of PTC, including 22 cPTC samples, 11 dsvPTC, 5 fvPTC, 1 svPTC, $1 \mathrm{fvPTC} / \mathrm{svPTC}, 1 \mathrm{cPTC} / \mathrm{fvPTC}$ and $2 \mathrm{cPTC} / \mathrm{fvPTC} / \mathrm{svPTC}$. The fusions included 20 RET/PTC1 (CCDC6-RET), 1 RET/PTC2 (PRKAR1A-RET), 8 RET/PTC3 (NCOA4-RET), 2 SPECC1L-RET, 1 EML4-RET, 1 TRIM24-RET, 1 CCDC186-RET, 2 TPR-NTRK1, 1 IRF2BPNTRK1, 1 SQSTM1-NTRK1 and 5 ETV6-NTRK3.

Of note, 4 additional potential kinase activating in-frame fusions (2 TFG-MET, 1 TGFGFR1 and 1 PRKD2-BRAF) and 3 mutations (1 BRAF p.T599del and 2 TSHR p.M453T and p.D633Y) were identified by the more comprehensive CSTP/CCFP panel. In addition, we found mutations associated with increased risk of thyroid cancer: 3 cases harbored alterations of $A P C$ ( 3 cmvPTC) and 2 cases harbored biallelic mutations of DICERl (1 fvPTC and 1 FTC). The 12 cases above were classified as indeterminate due to their low prevalence and uncertain molecular category. All genetic drivers found in this study are listed in Supplementary Table 2. 
The genetic alterations and correlation with clinicopathologic characteristics and outcomes are summarized in Table 2. Several strong associations between the covariates and mutation categories were observed. When comparing the 4 categories of mutation status: indeterminate, $R A S$-mutant, BRAF-mutant, and RET/NTRK fusion, all variables were found to be statistically significant ( $\mathrm{p}$-value $<0.05$ ), except for sex and AJCC T (tumor size) category. There were very few $R A S$-mutant samples, therefore we restricted analysis and comparison to $B R A F$-mutant and $R E T / N T R K$ fusions. After restricting the sample to those with $B R A F$-mutant vs $R E T / N T R K$ fusion status, statistically significant associations were still found among age, AJCC N (lymph node metastasis) and M (distant metastasis, all pulmonary) categories as well as remission at 1-year (pvalue $<0.05)$. Significantly, no distant metastasis was detected in any patients with $B R A F$-mutant thyroid tumors. Thirty-seven percent of patients with RET/NTRK fusion tumors did not attain remission at one year compared to less than $5 \%$ of patients with a tumor harboring a $B R A F$ mutation.

cPTC variant was the most common histology for both $B R A F$-mut $(\mathrm{n}=22)$ and $R E T / N T R K$ fusion subgroups ( $\mathrm{n}=22 ; 16 R E T, 3 N T R K 1,3 N T R K 3$ fusions). Even within this histological variant, we observed significant differences in metastatic behavior and remission status between these molecular subgroups. Metastases to lateral neck lymph nodes were found in 4/22 (18.2\%) of patients with a $B R A F$ mutation and 13/22 (59.1\%) of patients with RET/NTRK fusions. Distant metastasis was present in $8 / 22(36.4 \%)$ of the $R E T / N T R K$ fusion subgroup and none of the patients within $B R A F$-mut subgroup. Moreover, persistent disease at 1-year was more frequent in the subgroup harboring $R E T / N T R K$ fusions $(5 / 15 ; 33.3 \%)$ than those with mutations in $B R A F(1 / 18$; 5.6\%) (Supplementary Table 3). 
Consistent with previously published results, a greater percentage of pediatric patients presented with nodal and distant metastasis compared to adult tumors reported in the TCGA database (Figure 2). ${ }^{15}$ In adult DTC samples, lateral lymph node metastasis was slightly increased in patients with $R E T / N T R K$ fusions vs $B R A F$-mut, but the difference is less pronounced than in our pediatric population (Figure 2). By contrast, adults harboring $R A S$-mutant PTC rarely present with lymph node or distant metastasis. ${ }^{16}$ This also seems to be the case for pediatric patients with $R A S$-mutant DTC, although the significance of this is unknown due to the limited number of $R A S$ mutant DTCs in our cohort.

RET/NTRK fusions were more common in our cohort of patients less than 10 years old compared to patients $>10$ years old, as previously reported. ${ }^{13}$ Ten out of $11(91 \%)$ patients $<10$ years harbored fusion events. The prevalence gradually decreased in pediatric patients older than 10 years $(27 \%)$ and into adulthood (9\%). In contrast, only 1 BRAF p.V600E mutation (9\%) was

found among patients $<10$ years old compared to 25 pediatric patients $\geq 10$ years of age $(20 \%)$ with further increased prevalence in adult patients with $B R A F$-mutations where $58 \%$ of PTC in adults harboring a $B R A F$-mutation (Table 1 and Supplementary Figure 2A).

\section{DISCUSSION}

The Thyroid Cancer Genome Atlas (TCGA) reported that classification of adult PTC into molecularly-defined groups, $R A S$-like and $B R A F$-like, more accurately reflects cellular signaling, cellular differentiation and clinical behavior when compared to histology alone. ${ }^{1}$ Our data suggests that separating pediatric PTC with BRAF p.V600E mutations from PTC with RET/NTRK fusions more closely aligns with clinicopathological features with $B R A F$ positive PTC less common with decreased age and RET/NTRK fusion positive PTC more commonly associated with lateral neck 
(N1b) and distant (M1, pulmonary) metastasis. Both BRAF and RET/NTRK fusions were identified in cPTC, however, even within the same pathological variant, the molecular driver more accurately predicted metastatic behavior (Supplementary Table 3). This observation is in keeping with previous reports in adults ${ }^{1,2,11}$ and pediatrics ${ }^{12,13}$ that reclassification of thyroid cancers into genetic and molecular subtypes provides an opportunity for better informed clinical management compared to pathological classification alone. RAS-mutants showed similar predictability, associated with reduced metastatic behavior in our pediatric cohort compared to published data in adults, however, the low incidence of $R A S$-mutants in our cohort prevented clinically relevant statistical analysis.

There are differences in the composition of genetic variants in the pediatric population when compared to the adult. The incidence of a $B R A F$ p.V600E mutation in pediatric patients with PTC is lower and there is a lower risk for $B R A F$-associated widely invasive disease with decreased radioactive iodine avidity. ${ }^{5,12,13}$ In addition, coexisting mutations in the TERT promoter, TP53 and genes encoding effectors of the PI3K pathway $(P I K 3 C A)$ are frequent in $B R A F$-mut $(\sim 10 \%)$ and RET/NTRK ( 2.5\%) advanced adult thyroid tumors. ${ }^{1}$ In our cohort of pediatric tumors screened by the CSTP panel ( $\mathrm{n}=66)$, we did not find any coexisting mutations in $B R A F$-mut or $R E T / N T R K$ tumors. This suggests that with increasing age other age-related host factors may result in the accumulation of additional genetic alterations that negatively influence differentiation, response to therapy, and, subsequently, disease-specific morbidity and mortality supporting separation of how oncogenic landscape data is interpreted and incorporated into clinical practice for pediatric versus adult patients with DTC.

The availability of the CSTP has provided us with a wider lens in which to view pediatric thyroid carcinoma. The incorporation of a comprehensive cancer gene panel lowered the number 
of samples without an identifiable alteration and led to discovery of several findings that warrant further investigation. We demonstrate that $50 \%$ of tumors with distal metastasis had indeterminate drivers utilizing the miRInform panel. By contrast, only $30 \%$ of tumors characterized with the more comprehensive CSTP panel had indeterminate drivers and within these 20 samples, 12 harbored mutations that likely have an important role in thyroid tumorigenesis. These 12 cases were included in the indeterminate subgroup due to their relatively low prevalence and uncertain molecular category. These included fusions PRKD2-BRAF, TFG-MET (n=2) and TG-FGFR1 and mutations TSHR p.M453T and p.D633Y and BRAF p.T599del), all previously reported in thyroid tumors with exception of the novel PRKD2-BRAF fusion (Supplementary Table 2). ${ }^{117-19}$ Future studies are underway to more clearly define the influence of these genetic alterations on altered signaling pathways and thyroid cell differentiation.

The observation of a higher incidence of RET/NTRK fusions as well as their association with more metastatic behavior compared to $B R A F$ in our pediatric cohort emphasizes the importance of expanding our knowledge of the pediatric DTC molecular landscape. In the adultbased TCGA, analysis revealed a fairly quiet adult PTC genome allowing for a more precise evaluation of the effects of the genetic drivers on signaling pathway activation and differentiation. In the TCGA, a 71-gene signature generated by comparison of $B R A F$-mut and $R A S$-mut tumors was used to construct a $B R A F^{\mathrm{V} 600 \mathrm{E}}-R A S$ score $(\mathrm{BRS})$ that separated tumors based on MAPK pathway output and clinical behavior. In the TCGA analysis, NTRK1/3 fusions were largely neutral and virtually all $R E T$ fusions were only weakly $B R A F$-like. ${ }^{1}$ The $R E T$-fusion subgroup in these adult tumors also exhibited an intermediate Thyroid Differentiation Score (TDS; 16-gene signature including thyroid specific genes such as $S L C 5 A 5, T G, T P O, P A X 8, T S H R$ and others), lower than the well differentiated $R A S$-mut $(H / K / N R A S$ and $P A X 8 / P P A R G)$ tumors and higher than the $B R A F$ - 
mut (BRAF p.V600E; Supplementary Figure 2). Considering the high prevalence of RET/NTRK fusions in pediatric DTC, and their association with more metastatic behavior, it will be crucial to generate the transcriptional signatures of $R E T / N T R K$ and $B R A F$-mutant subgroups in the pediatric population to understand the differential impact of these alterations on signaling pathways, differentiation and clinical outcomes.

There are several limitations to this study, supporting the need for multi-center, prospective studies to expand our knowledge on the potential use of genetic and molecular analysis for stratification of therapy. The sample size is relatively small at 131 samples, especially as the analysis was divided between the miRInform and CSTP samples at 65 and 66 samples, respectively. It is also worth repeating that $R E T / N T R K$ prevalence in this study may be underestimated as the miRInform panel did not include analysis for NTRK1, NTRK3, BRAF and $A L K$ fusions as well as expanded $R E T$ fusion isoforms. Unfortunately, as previously stated, the samples initially analyzed by the miRInform panel were not available for reanalysis using the CSTP. Last, our conclusions are only based on oncogenic driver alterations. Additional studies are ongoing to define the differentiation-score based on multiplatform analysis including RNA and microRNA expression.

An important strength of our study is that many of these oncogenic events that are more common in pediatrics compared to adults with DTC can now be targeted with FDA-approved agents. Of particular note is the identification of RET and NTRK fusions and positive association of these events with persistent disease and metastatic spread. Larotrectinib (NTRK inhibitor) and selpercatinib (RET inhibitor) have shown dramatic efficacy in clinical trials in both solid tumors and hematologic malignancies, including a limited number of thyroid tumors harboring NTRK and $R E T$ fusions included in these studies. Significantly, these studies have shown durable responses 
in a multitude of patients with limited adverse events. ${ }^{20,21}$ Interestingly, larotrectinib was shown to induce partial response and restore iodine uptake in one case of RAI refractory adult thyroid cancer harboring the EML4-NTRK3 fusion ${ }^{22}$ opening the way for similar therapies in pediatric tumors where these alterations are frequent and often associated with poor prognosis.

\section{CONCLUSIONS}

The combined dataset used in this study represents an evolutionary change in the information gained from genetic and molecular analysis over the span of a few short years. Based on our data, categorizing pediatric DTC into $R A S$-mutant, $B R A F$-mutant, and $R E T / N T R K$ fusion variants more accurately separates the higher risk of invasive behavior for RET/NTRK fusion driven PTC compared to PTC harboring BRAF p.V600E mutations. RET/NTRK fusion tumors metastasize to lateral neck lymph nodes at a significantly higher frequency than $B R A F$ p.V600E PTC. Further, we did not observe distant metastasis in any patients with BRAF p.V600E mutations. These findings support the incorporation of somatic cancer gene analysis to improve the diagnostic accuracy for FNA as well as the potential utility to incorporate oncogenic data to stratify the surgical approach and to identify tumors that may benefit from oncogene-specific systemic therapies. Additional studies are underway to define the differences in differentiation score between BRAF p.V600E and RET/NTRK fusion PTC, differences between pediatric and adults PTC with the same oncogenic alterations, as well as to confirm the tumorigenic potential of the novel-alterations identified using the comprehensive CSTP panel. 


\section{DECLARATIONS}

Ethics approval and consent to participate: The study was approved by CHOP's Institutional Review Board.

\section{Consent for publication:}

\section{Availability of data and material:}

\section{Competing Interests:}

Author Disclosure Statement: TWL has consulted for Novartis, Cellectis, Bayer, Loxo, Lilly, Deciphera, Jumo Health, and Y-mAbs Therapeutics and has research funding from Pfizer and Bayer.

Funding: This work was supported in part by a grant from NIH R01CA21451 (ATF), The Children's Hospital of Philadelphia Frontier Programs (ATF, JCRF, AI, SMM, LS, ER, AJB), and the Children's Hospital of Philadelphia Foerderer Grant (JCRF)

\section{Corresponding Author}

Andrew J Bauer, MD

The Thyroid Center

Division of Endocrinology and Diabetes, The Children's Hospital of Philadelphia, 3500 Civic Center Boulevard, Buerger Center 12-149, Philadelphia, PA 19104

Tel: 215-590-5129

Email: bauera@chop.edu 


\section{REFERENCES}

1. Cancer Genome Atlas Research N: Integrated genomic characterization of papillary thyroid carcinoma. Cell 159:676-90, 2014

2. Krasner JR, Alyouha N, Pusztaszeri M, et al: Molecular mutations as a possible factor for determining extent of thyroid surgery. J Otolaryngol Head Neck Surg 48:51, 2019

3. Labourier E, Fahey TJ, 3rd: Preoperative molecular testing in thyroid nodules with Bethesda VI cytology: Clinical experience and review of the literature. Diagn Cytopathol, 2020

4. Bauer AJ: Molecular Genetics of Thyroid Cancer in Children and Adolescents. Endocrinol Metab Clin North Am 46:389-403, 2017

5. Paulson VA, Rudzinski ER, Hawkins DS: Thyroid Cancer in the Pediatric Population. Genes (Basel) 10, 2019

6. Mostoufi-Moab S, Labourier E, Sullivan L, et al: Molecular Testing for Oncogenic Gene Alterations in Pediatric Thyroid Lesions. Thyroid 28:60-67, 2018

7. Lloyd R, Osamura R, Klöppel G, et al: WHO classification of tumours of endocrine organs [Internet]. WHO IARC; 2017 [cited 2018 Apr 27]. 355 p.(IARC WHO Classification of Tumours (Book 10); vol. 10),

8. Edge SB, Compton CC: The American Joint Committee on Cancer: the 7th edition of the AJCC cancer staging manual and the future of TNM. Ann Surg Oncol 17:1471-4, 2010

9. Surrey LF, MacFarland SP, Chang F, et al: Clinical utility of custom-designed NGS panel testing in pediatric tumors. Genome Med 11:32, 2019

10. Chang F, Lin F, Cao K, et al: Development and Clinical Validation of a Large Fusion Gene Panel for Pediatric Cancers. J Mol Diagn 21:873-883, 2019

11. Yip L, Nikiforova MN, Yoo JY, et al: Tumor genotype determines phenotype and disease-related outcomes in thyroid cancer: a study of 1510 patients. Ann Surg 262:519-25; discussion 524-5, 2015

12. Bauer AJ: Pediatric Thyroid Cancer: Genetics, Therapeutics and Outcome. Endocrinol Metab Clin North Am 49:589-611, 2020

13. Pekova B, Sykorova V, Dvorakova S, et al: RET, NTRK, ALK, BRAF, and MET Fusions in a Large Cohort of Pediatric Papillary Thyroid Carcinomas. Thyroid, 2020

14. Potter SL, Reuther J, Chandramohan R, et al: Integrated DNA and RNA sequencing reveals targetable alterations in metastatic pediatric papillary thyroid carcinoma. Pediatr Blood Cancer 68:e28741, 2021

15. Zimmerman D, Hay ID, Gough IR, et al: Papillary thyroid carcinoma in children and adults: long-term follow-up of 1039 patients conservatively treated at one institution during three decades. Surgery 104:1157-66, 1988

16. Kakarmath S, Heller HT, Alexander CA, et al: Clinical, Sonographic, and Pathological Characteristics of RAS-Positive Versus BRAF-Positive Thyroid Carcinoma. J Clin Endocrinol Metab 101:4938-4944, 2016

17. Pfeifer A, Rusinek D, Zebracka-Gala J, et al: Novel TG-FGFR1 and TRIM33NTRK 1 transcript fusions in papillary thyroid carcinoma. Genes Chromosomes Cancer 58:558566,2019

18. Pozdeyev N, Gay LM, Sokol ES, et al: Genetic Analysis of 779 Advanced Differentiated and Anaplastic Thyroid Cancers. Clin Cancer Res 24:3059-3068, 2018 
19. Schulten HJ, Salama S, Al-Mansouri Z, et al: BRAF mutations in thyroid tumors from an ethnically diverse group. Hered Cancer Clin Pract 10:10, 2012

20. Ortiz MV, Gerdemann U, Raju SG, et al: Activity of the Highly Specific RET Inhibitor Selpercatinib (LOXO-292) in Pediatric Patients With Tumors Harboring RET Gene Alterations. JCO Precis Oncol 4, 2020

21. Wirth LJ, Sherman E, Robinson B, et al: Efficacy of Selpercatinib in RET-Altered Thyroid Cancers. N Engl J Med 383:825-835, 2020

22. Groussin L, Clerc J, Huillard O: Larotrectinib-Enhanced Radioactive Iodine Uptake in Advanced Thyroid Cancer. N Engl J Med 383:1686-1687, 2020 
Table 1. Demographics of 131 pediatric and adolescent patients.

\begin{tabular}{lccccc}
\hline & n (\%) & Indeterminate & $\boldsymbol{R} \boldsymbol{A}$ S-mut & BRAF-mut $\boldsymbol{R E T}$ /NTRK \\
\hline Sex & & & & & \\
$\quad$ Female & $97(74 \%)$ & $40(75.5 \%)$ & $5(55.6 \%)$ & $22(84.6 \%)$ & $30(69.8 \%)$ \\
$\quad$ Male & $34(26 \%)$ & $13(24.5 \%)$ & $4(44.4 \%)$ & $4(15.4 \%)$ & $13(30.2 \%)$ \\
Age & & & & & \\
$\quad<10$ & $11(8.4 \%)$ & $0(0 \%)$ & $0(0 \%)$ & $1(3.8 \%)$ & $10(23.3 \%)$ \\
$>=10$ & $120(91.6 \%)$ & $53(100 \%)$ & $9(100 \%)$ & $25(96.2 \%)$ & $33(76.7 \%)$ \\
Panel & & & & & \\
$\quad$ miRInform & $65(49.6 \%)$ & $33(62.3 \%)$ & $8(88.9 \%)$ & $12(46.2 \%)$ & $12(27.9 \%)$ \\
CSTP/CCFP & $66(50.4 \%)$ & $20(37.7 \%)$ & $1(11.1 \%)$ & $14(53.8 \%)$ & $31(72.1 \%)$ \\
Diagnosis & & & & & \\
$\quad$ cPTC & $60(45.8 \%)$ & $15(28.3 \%)$ & $1(11.1 \%)$ & $22(84.6 \%)$ & $22(51.2 \%)$ \\
$\quad$ fvPTC & $29(22.1 \%)$ & $18(33.9 \%)$ & $5(55.6 \%)$ & $1(3.8 \%)$ & $5(11.6 \%)$ \\
$\quad d s v P T C$ & $14(10.7 \%)$ & $3(5.7 \%)$ & $0(0 \%)$ & $0(0 \%)$ & $11(25.6 \%)$ \\
$\quad$ cPTC/fvPTC & $8(6.1 \%)$ & $5(9.4 \%)$ & $0(0 \%)$ & $2(7.7 \%)$ & $1(2.3 \%)$ \\
FTC & $9(6.9 \%)$ & $7(13.2 \%)$ & $2(22.2 \%)$ & $0(0 \%)$ & $0(0 \%)$ \\
Other* & $11(8.4 \%)$ & $5(9.4 \%)$ & $1(11.1 \%)$ & $1(3.8 \%)$ & $4(9.3 \%)$ \\
\hline
\end{tabular}

*Other includes solid-variant PTC (svPTC), cribriform-morular variant PTC (cmvPTC), Warthin-like PTC (wlPTC) and mixed histologies. 


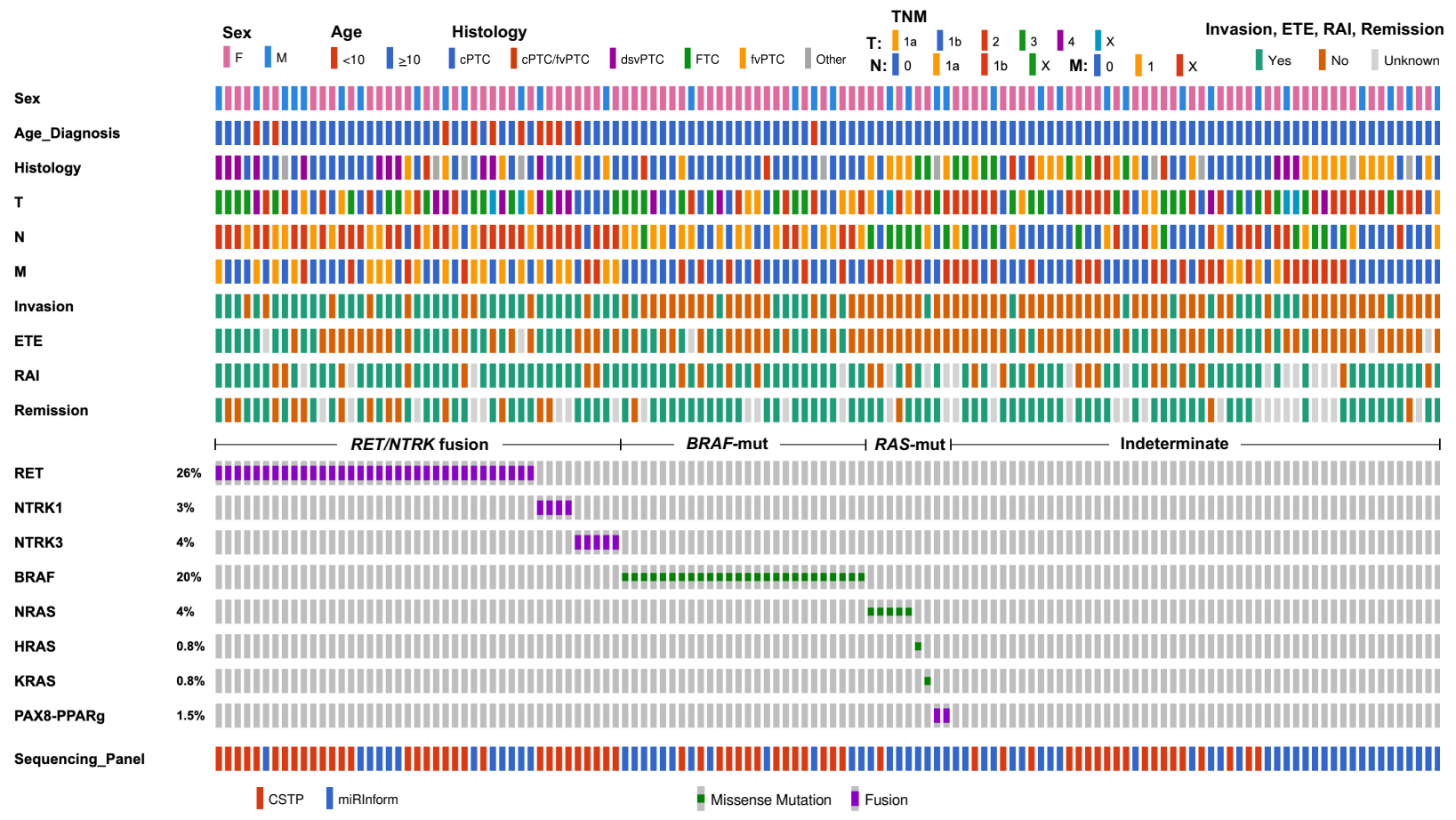

Figure 1. Genetic landscape and clinicopathological characteristics of 131 pediatric thyroid cancers. Clinicopathological characteristics includes sex, age, histology, tumor (T) status, lymph node $(\mathrm{N})$ metastasis status, distant metastasis status $(\mathrm{M})$, invasion, extrathyroidal extension (ETE), radioactive iodine (RAI) therapy and remission. The most frequent genetic alterations (fusion oncogenes and mutations) and their prevalence are shown. Genetic alterations were categorized in $R E T / N T R K$ fusions (RET and NTRK1/3 fusions), BRAF-mut (BRAF p.V600E) and RAS-mut (H$K-N R A S$ and $P A X 8 / P P A R G)$. 
Table 2. Association between the covariates and mutations categories: $B R A F$-mut, $R A S$-mut, RET/NTRK fusion and Indeterminate.

\begin{tabular}{|c|c|c|c|c|c|c|c|}
\hline & & Indeterminate & $R A S$-mut & $B R A F$-mut & RET/NTRK & $\begin{array}{l}\text { p-value, } \\
4 \text { Categories }\end{array}$ & $\begin{array}{l}\text { p-value, } \\
B R A F \text { vs } \\
\text { RET/NTRK }\end{array}$ \\
\hline \multirow{2}{*}{ ETE } & Yes & $13(24.5 \%)$ & $0(0 \%)$ & $12(46.2 \%)$ & $23(53.5 \%)$ & \multirow{2}{*}{$2.66 \mathrm{E}-03$} & \multirow{2}{*}{ 8.03E-01 } \\
\hline & No & $40(75.5 \%)$ & $9(100 \%)$ & $14(53.8 \%)$ & $20(46.5 \%)$ & & \\
\hline \multirow{2}{*}{ Invasion } & Yes & $11(20.8 \%)$ & $1(11.1 \%)$ & $8(30.8 \%)$ & $34(79.1 \%)$ & \multirow{2}{*}{ 2.13E-08 } & \multirow{2}{*}{$1.28 \mathrm{E}-04$} \\
\hline & No & $42(79.2 \%)$ & $8(88.9 \%)$ & $18(69.2 \%)$ & $9(20.9 \%)$ & & \\
\hline \multirow{2}{*}{ Histology } & FTC & $7(13.2 \%)$ & $2(22.2 \%)$ & $0(0 \%)$ & $0(0 \%)$ & \multirow{2}{*}{ 5.37E-03 } & \multirow{2}{*}{$1.00 \mathrm{E}+00$} \\
\hline & PTC & $46(86.8 \%)$ & $7(77.8 \%)$ & $26(100 \%)$ & $43(100 \%)$ & & \\
\hline \multirow{2}{*}{ Remission } & Yes & $35(94.6 \%)$ & $6(85.7 \%)$ & $20(95.2 \%)$ & $22(62.9 \%)$ & \multirow{2}{*}{ 8.74E-04 } & \multirow{2}{*}{$9.08 E-03$} \\
\hline & No & $2(5.4 \%)$ & $1(14.3 \%)$ & $1(4.8 \%)$ & $13(37.1 \%)$ & & \\
\hline \multirow{2}{*}{ Sex } & Female & $40(75.5 \%)$ & $5(55.6 \%)$ & $22(84.6 \%)$ & $31(72.1 \%)$ & \multirow{2}{*}{$3.70 \mathrm{E}-01$} & \multirow{2}{*}{$3.75 \mathrm{E}-01$} \\
\hline & Male & $13(24.5 \%)$ & $4(44.4 \%)$ & $4(15.4 \%)$ & $12(27.9 \%)$ & & \\
\hline \multirow{2}{*}{ Age } & $<10$ & $0(0 \%)$ & $0(0 \%)$ & $1(3.8 \%)$ & $10(23.3 \%)$ & \multirow{2}{*}{ 5.98E-03 } & \multirow{2}{*}{$3.75 \mathrm{E}-01$} \\
\hline & $\geq 10$ & $53(100 \%)$ & $9(100 \%)$ & $25(96.2 \%)$ & $33(76.7 \%)$ & & \\
\hline \multirow{3}{*}{ M } & 0 & $27(50.9 \%)$ & $2(22.2 \%)$ & $20(76.9 \%)$ & $19(44.2 \%)$ & \multirow{3}{*}{$5.86 \mathrm{E}-06$} & \multirow{3}{*}{ 1.92E-04 } \\
\hline & 1 & $4(7.5 \%)$ & $1(11.1 \%)$ & $0(0 \%)$ & $18(41.9 \%)$ & & \\
\hline & $\mathrm{X}$ & $22(41.5 \%)$ & $6(66.7 \%)$ & $6(23.1 \%)$ & $6(13.9 \%)$ & & \\
\hline \multirow{4}{*}{$\mathrm{N}$} & 0 & $28(52.8 \%)$ & $2(22.2 \%)$ & $7(26.9 \%)$ & $3(7.0 \%)$ & \multirow{4}{*}{ 4.09E-09 } & \multirow{4}{*}{ 1.03E-04 } \\
\hline & $1 \mathrm{a}$ & $7(13.2 \%)$ & $1(11.1 \%)$ & $14(53.8 \%)$ & $11(25.6 \%)$ & & \\
\hline & $1 b$ & $9(17.0 \%)$ & $0(0.0 \%)$ & $4(15.4 \%)$ & $29(67.4 \%)$ & & \\
\hline & $\mathrm{X}$ & $9(17.0 \%)$ & $6(66.7 \%)$ & $1(3.8 \%)$ & $0(0 \%)$ & & \\
\hline \multirow{6}{*}{$\mathrm{T}$} & $1 \mathrm{a}$ & $4(7.5 \%)$ & $2(22.2 \%)$ & $4(15.4 \%)$ & $4(9.3 \%)$ & \multirow{6}{*}{$1.12 \mathrm{E}-01$} & \multirow{6}{*}{$8.70 \mathrm{E}-01$} \\
\hline & $1 b$ & $8(15.1 \%)$ & $1(11.1 \%)$ & $7(26.9 \%)$ & $10(23.3 \%)$ & & \\
\hline & 2 & $24(45.3 \%)$ & $4(44.4 \%)$ & $5(19.2 \%)$ & $6(14.0 \%)$ & & \\
\hline & 3 & $13(24.5 \%)$ & $1(11.1 \%)$ & $8(30.8 \%)$ & $14(32.6 \%)$ & & \\
\hline & 4 & $2(3.8 \%)$ & $0(0 \%)$ & $2(7.7 \%)$ & $7(16.3 \%)$ & & \\
\hline & $\mathrm{X}$ & $2(3.8 \%)$ & $1(11.1 \%)$ & $0(0 \%)$ & $2(4.7 \%)$ & & \\
\hline
\end{tabular}



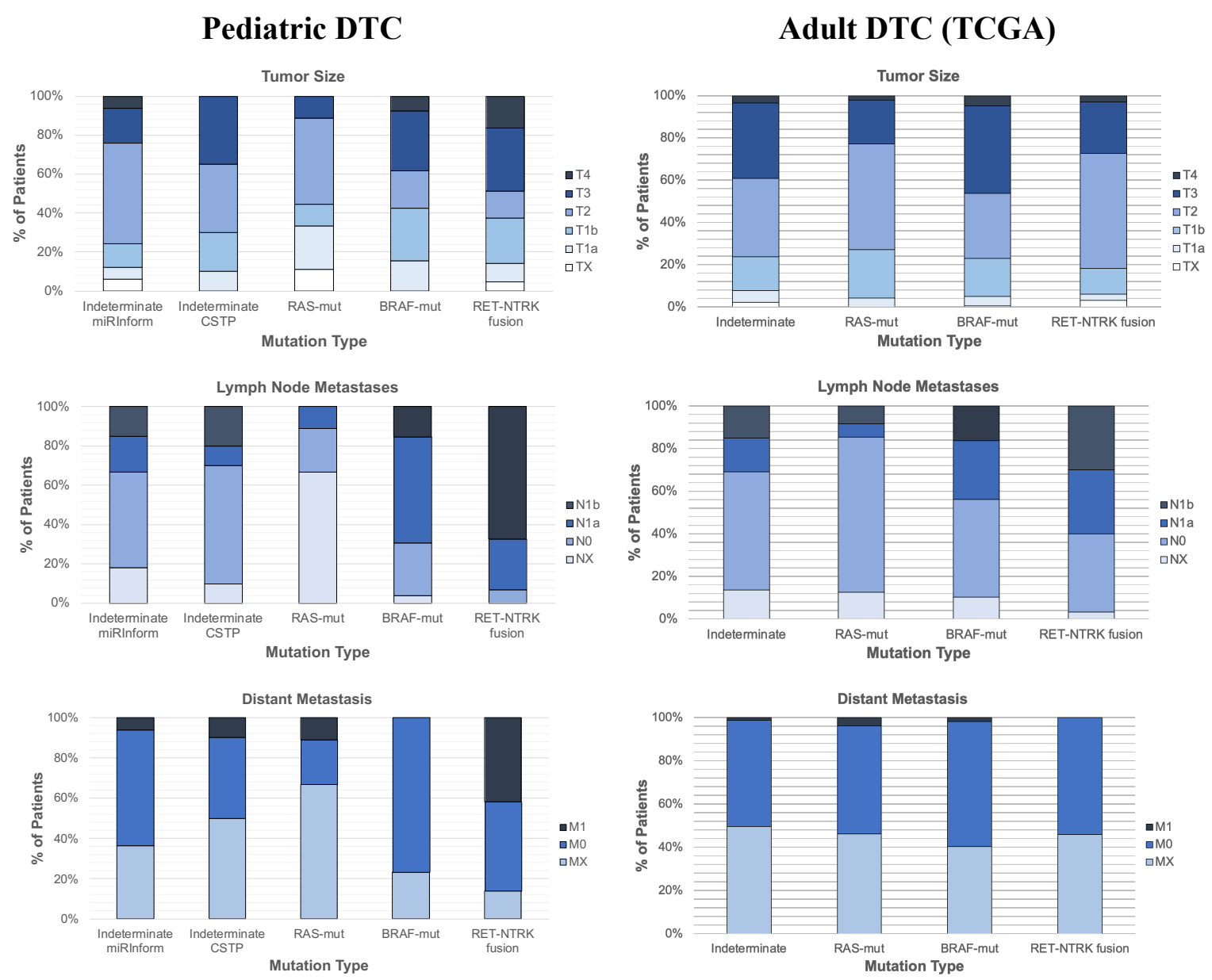

Figure 2. TNM staging of pediatric and adult thyroid cancers according to mutational status. Tumor Size, Lymph Node Metastasis and distant metastasis classification of pediatric differentiated thyroid cancer $(\mathrm{n}=131$; combined CSTP and miRInform samples) and adult differentiated thyroid cancers ( $\mathrm{n}=496$; TCGA) categorized by Indeterminate, $R A S$-mut, $B R A F$ mut, and RET/NTRK fusion variants. 